\title{
A telehealth-based training model for an ambulatory care pharmacy skills course
}

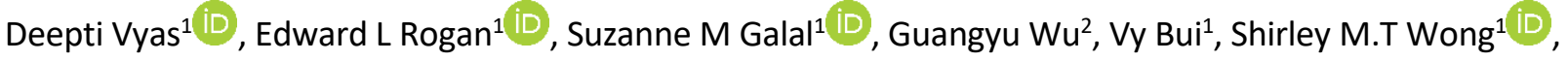 \\ Sabrina $\mathrm{Wu}^{1}$ \\ ${ }^{1}$ Department of Pharmacy practice, Thomas J. Long School of Pharmacy, University of the Pacific, United States \\ ${ }^{2}$ Fu Foundation School of Engineering and Applied Science, Columbia University, United States
}

\author{
Keywords \\ Ambulatory care skill \\ Covid-19 \\ Learning \\ Professional communication skill \\ Remote \\ Simulation \\ Telehealth \\ Correspondence \\ Deepti Vyas \\ Department of Pharmacy practice \\ University of the Pacific \\ Thomas J. Long School of Pharmacy \\ United States \\ vyasd80@gmail.com
}

\begin{abstract}
Description: Increasingly, pharmacy services are provided using telehealth-based modalities. This paper describes a pharmacy skills course that utilised telehealth principles to train students on the technical and communications skills necessary for the ambulatory care setting. Zoom breakout rooms, electronic health records, YouTube video vignettes, and teaching assistants portraying patients/physicians simulated a telehealth-based ambulatory care setting. Evaluation: Five quizzes and six written assignments were utilised to measure student's knowledge and skills. At the end of the course, students were evaluated through a three-station objective structured clinical exam (OSCE). Students also completed a pre/post attitudes survey. Result: Overall, students performed well on various assessments including quizzes and written assignments. The majority of the students performed well on the OSCE. Significant improvement was noted on all items in the attitudes survey. Conclusion: This study suggests that a telehealth training model can be effective in teaching pharmacy students both the technical and communication skills necessary for practice in the ambulatory care setting.
\end{abstract}

\section{Introduction}

Telehealth is the use of electronic data and telecommunications technologies to provide care to patients and collaborate with other healthcare professionals who are in remote locations (American Telemedicine Association, 2017). Telehealth expands access to care for those patients who are in rural areas, unable to access care due to disabilities, or need specialised care that is unavailable in their immediate vicinity.

Telehealth technologies such as videoconferencing are increasingly used to provide pharmacy services, especially in the advent of Covid-19 (Yemm et al., 2020). Pharmacists need to be able to manage patients who are located in remote locations using both realtime interactions and remote monitoring tools such as home blood pressure, blood glucose readings, food logs, and international normalised ratio (INR) results, among others (Klug et al., 2011; Traynor, 2013; Perdew et al., 2017; Badowski et al., 2018; Litke et al., 2018).

A thorough understanding of available technologies and verbal/non-verbal communication techniques is imperative for the telehealth pharmacist to build a relationship with patients in the virtual environment. In addition, requesting and retrieving supplemental data such as laboratory tests, imaging, and other health provider findings is crucial in providing adequate care in the telehealth-based environment.

The recent survey presented in the International Pharmaceutical Federation (FIP) report showed that a large number of pharmacy schools do not offer training on digital health (FIP, 2021). The FIP development Goal 20 recommends that pharmacy programmes develop courses, which facilitate the development of a digitally literate pharmacy workforce. Education on telehealth, mobile applications, wearable technology, and electronic health records (EHR) is imperative so that 
pharmacy graduates are competent and can serve as patient resources in the digital era (FIP development goals). It is clear that pharmacy students must receive training on digital health technologies so that they can be ready for contemporary practice models and the potential for expanded telehealth services in the future.

At the University of the Pacific, School of Pharmacy, the new core curriculum includes five skills courses, which focus on various practice-specific skills. Of these, the Skills III course emphasised technical skills and contemporary communication techniques used in the ambulatory care setting. Covid-19 necessitated the faculty to recreate the course using virtual platforms. In doing so, creative strategies gave way to the use of telehealth-based principles for modelling and delivering training on the technical skills and communication techniques used in the ambulatory care setting. This allowed for active immersion experiences and opportunities to provide one on one feedback. The purpose of this paper is to describe the components of this course and its impact on student knowledge, skills and attitudes.

\section{Description of course}

First year Doctor of Pharmacy students who enrolled in the Skills III course at the University of the Pacific, school of pharmacy were eligible to participate in the study. This course is a required core course in the accelerated curriculum and occurs in the third semester of the six-semester didactic curriculum. Besides the Skills III course, these students are also enrolled in two therapeutics courses focused on Cardiovascular diseases (three-credit-hour course) and Endocrine diseases (One-credit-hour). In addition, these students had completed a two-credit hour course focused on self-care course in the prior spring term.

The learning objectives of the course were 1) Communicate effectively with patients and other healthcare professionals; 2) Use the Pharmacists Patient Care Process (PPCP) to conduct a patient interview, to collect pertinent patient information and then develop an assessment and plan; 3) Collect, assess, and address factors leading to medication non-adherence; 4) Utilise motivational interviewing techniques to address medication non-adherence; 5) Complete a medication reconciliation and relay recommendations to another healthcare professional; 6) Teach and assess proper device technique and patient understanding; 7) Provide patient counselling on common prescription and nonprescription therapy; and 8) Address vaccine hesitancy using motivational interviewing techniques.
This study was deemed exempt by the University institutional review board.

\section{Teaching assistant training}

Forty-five teaching assistants (TAs) were recruited from the second year class as well as the graduate TA pool. A TA course was developed to train TAs to portray a patient/physician within a character biography and script. An example of a character biography and script can be found in Appendix 1. TAs were also trained to provide feedback using a standardised rubric created by course faculty. TAs met on a weekly basis for an hour to receive training. Course faculty modelled a telehealth interaction for each simulation and posted it on YouTube for the TAs to review prior to class. TAs were then provided three scripts and character biographies which provided background information on the patient's mood, home life, and other relevant information to allow the TA to understand the role/s they were expected to portray each week. During the TA class, faculty provided a ten-minute lecture and then utilised zoom breakout rooms to allow TAs to role-play each script and practice providing constructive feedback.

\section{Class format}

Students met for two hours per week for 14 weeks, with most weeks being synchronous. Asynchronous sessions were used to deliver recorded lectures on topics that required a preamble prior to the simulation. Asynchronous lecture topics included 1) Patient adherence; 2) Motivational interviewing; 3) Medication reconciliation/transitions of care; 4) Drug related problems (DRPs); and 5) Vaccine hesitancy. Prior to each synchronous session, students watched a YouTube video that demonstrated the proper technique for that week's simulation.

Students also received three links to patient charts on EHRGO, an educational EHR programme, and were asked to review all three cases prior to the synchronous simulations. The EHR contained demographic information about the patient, pertinent laboratory data, previous provider notes, diagnostic test results, and the patient's medication profile. Synchronous sessions began with a 10-15 minute lecture on the topic of the day and the agenda for the session. Students were then divided into groups of three and assigned to a zoom breakout room with a TA.

Students were asked to download a specific zoom background (clinic room), so that the videoconference mimicked a professional environment and created a sense of shared space for the students. Each student completed one consult while the other two listened 
and documented the care of the patient. At the end of each consult, the TA provided feedback on the student's communication skills and brainstormed areas of improvement.

For certain simulations, all three students discussed the cases first and then completed individual consults. A stepwise approach was utilised for the course, so that the course started with simpler exercises and gradually delved into more complex clinical scenarios that required therapeutic knowledge in addition to professional communication skills. Cases were also coordinated with current therapeutics classes to help reinforce knowledge and apply that information in a practice setting.

The course format included several main topics related to ambulatory care. Simulations were created to provide practice and feedback on each of these areas (Table I).

Table I: Course format

\begin{tabular}{|c|c|c|c|}
\hline Week & Topic & Activity/Assignment & Quiz \\
\hline Week 1 & Introduction to professional communication & Live lecture & $\mathrm{N}$ \\
\hline Week 2 & Interviewing a patient using the LOQQSAM tool & Simulation/case write-up & $\mathrm{Y}$ \\
\hline Week 3 & Assessing and addressing medication adherence & $\begin{array}{l}\text { Recorded lecture/ written reflection comparing } \\
\text { two patient consults addressing medication } \\
\text { adherence }\end{array}$ & Y \\
\hline Week 4 & Assessing and addressing medication adherence & Simulation/case write-up & $\mathrm{N}$ \\
\hline Week 5 & Drug related problems (DRPs) & Recorded lecture & $\mathrm{Y}$ \\
\hline Week 6 & $\begin{array}{l}\text { Identifying drug related problems in relation to a } \\
\text { patient's plan }\end{array}$ & Simulation/ SOAP note & $\mathrm{N}$ \\
\hline Week 7 & Pharmacist furnishing & Recorded lecture & $\mathrm{Y}$ \\
\hline Week 8 & Vaccine hesitancy/Managing difficult patients & Simulation/ video-log reflection & $\mathrm{N}$ \\
\hline Week 9 & Physical assessment techniques & Recorded lecture & $\mathrm{Y}$ \\
\hline Week 10 & Device teaching- inhalers & Simulation/ SOAP note & $\mathrm{N}$ \\
\hline Week 11 & DRPs and prescription drug counseling & Simulation & $\mathrm{N}$ \\
\hline Week 12 & Medication reconciliation and transitions of care & Simulation/ SOAP note & $\mathrm{N}$ \\
\hline Week 13 & OSCE & Three simulation stations & NA \\
\hline Week 14 & Device teaching- insulin and glucose meters & Recorded lecture & $\mathrm{N}$ \\
\hline
\end{tabular}

assessment and plan; $\mathrm{Y}=\mathrm{Yes} ; \mathrm{N}=\mathrm{No}$; OSCE= Objective structured clinical exam

The descriptions of each simulation are below:

\section{Interviewing a patient}

For this simulation, students interviewed the patient to obtain a complete history as well as use the LOQQSAM (location, onset, quality, quantity, setting, associated symptoms, and modifying factors) technique to determine the patient's suitability for self-care for a given chief complaint. Once the assessment was complete, students recommended and counselled the patient on an over-the-counter (OTC) medication for their ailment. Students then documented the interview using a patient history template. The objective of this session was to interview the patient, collect relevant information about the chief complaint, and recommend an OTC medication.

\section{Addressing medication adherence}

Addressing medication adherence is a complicated process that requires the ability to assess and evaluate all possible reasons for non-adherence that can include, but are not limited to, financial, educational, attitudinal, and cognitive issues. When the basis for non-adherence is determined, then an acceptable solution must be provided and accepted by the patient.

To prepare for this exercise, students first watched a YouTube video depicting two pharmacist consultations on medication adherence, with one consult being superior to the other. Students were then asked to submit a written reflection on their impression of the two consults and which they thought would be more effective and why. During the live simulation session, students first reviewed the patient's chart on EHRGO and medication refill history to determine any objective issues with non-adherence. Students then interviewed the TA portraying that same patient. Students had to conduct an interview and address the non-adherence in a non-judgmental and empathetic manner. Three scripts were developed for this simulation, with all three simulations requiring the use of motivational interviewing to determine the cause of the nonadherence and then to make recommendations to address the specific causes. Some reasons for nonadherence included complicated medication regimen, unawareness of the importance of taking medications as prescribed, language barriers, financial burden, depression, and forgetfulness. 


\section{Identifying drug related problems (DRP)}

This simulation portrayed a follow-up appointment that required the student to use the 3 Cs model (control, complications, and compliance) to interview the patient. Again, students first reviewed the information in the patient's chart on EHRGO, and then interviewed the patient to collect information about the 3 Cs. The interview and chart information was designed to reveal several DRPs, if done properly, that the student was expected to assess and address. For example, for case 1 , the DRP was constipation related to opioid use and uncontrolled blood pressure. For case 2 , the patient was taking two non-steroidal antiinflammatory drugs, was an active cigarette smoker, and needed therapy for gastroesophageal reflux disorder (GERD). For case 3, the sitagliptin dose needed to be renally adjusted, the patient was experiencing diarrhoea from excessive use of milk of magnesia, and the patient required alternative therapy for GERD. Students were required to put a plan together that would address the above DRPs. The TA's then led a discussion regarding the DRPs and proper plans for each patient using a discussion guide developed by the faculty.

\section{Addressing vaccine hesitancy/managing difficult patients}

Vaccine hesitancy is a major issue limiting the number of patients who are vaccinated and is a hindrance to the goals of the Centre for Disease Control (CDC) Healthy People 2020 initiative (CDC 2020). If given the correct tools, students can learn to address and dispel some of the major misinformation leading to vaccine hesitancy (Vyas et al.,2018). Students first watched a recorded lecture on vaccine hesitancy, which listed common myths and rebuttals. For the vaccine hesitancy simulation, TAs portrayed either a patient or the parent of a child. Each consult included at least two reasons for the vaccine hesitancy, and students were expected to address those concerns using motivational interviewing techniques. The simulation was similar to one previously described in the literature (Vyas et al.,2018).

\section{Physical assessment techniques}

Students at the school of pharmacy receive basic physical assessment training in the first semester of the curriculum with more advanced techniques slated for the Skills III course. Due to Covid-19, physical assessment has been postponed to a future semester. Instead, students received an asynchronous lecture on physical assessment techniques and their interpretation.

\section{Educating a patient on devices}

This simulation focused on assessing and correcting improper inhaler technique. One YouTube video demonstrating proper technique was created to refresh students on proper technique related to aerosolised inhalers (albuterol and Respimat) and dry powder inhalers (Advair Diskus). For the simulation, three YouTube videos were created showing a patient making several errors in their inhaler technique. These YouTube videos were used by the TA to demonstrate incorrect technique to the students as the TAs did not have actual inhalers at home. Students performed a follow-up interview with the TA that included a demonstration of their technique using the above videos. Students then assessed any errors of technique and/or compliance and counselled the TA on correct inhaler technique and adherence in a non-judgmental manner.

\section{Prescription drug counselling}

Students received three EHRGO patient charts ahead of the simulation day. Working in groups of three, each student first had to complete a patient interview to assess the patient's chief complaint. Then all three students discussed the case and developed an assessment and plan. The plan was then relayed to the patient orally, and the student entered an electronic prescription into the computerised provider order entry (CPOE) portal on EHRGO. Students were then instructed to switch gears and provide counselling on the drug they chose at the dispensing pharmacy. Each student had to use the three prime questions (what did the doctor tell you this medication is for, how did the doctor tell you to take this medication, and what did the doctor tell you to expect) to counsel the patient on the prescription that they entered into the CPOE. By having the students switch roles, the authors hoped to emphasise transitions of care from the ambulatory care setting to the dispensing pharmacy.

\section{Medication reconciliation}

For the medication reconciliation simulation, students watched a store and forward video that depicted a medication history interview and compared that interview with the medication profile on EHRGO. Students noted any discrepancies or inappropriate therapy and brainstormed their findings with their peers in the breakout rooms. After the discussion, students used the SBAR (situation, background, assessment, and recommendation) tool to relay their recommendations to the TA who portrayed an attending physician. Each case contained at least five discrepancies, with some being obvious such as dosing errors and the remaining related to more complex 
clinical issues such as disease contraindications, drugdrug interactions or renal dosing.

\section{Evaluation}

One hundred and ninety seven students participated in this course. Students completed six written assignments documenting their interaction with the patient. In general, written assignments were of high quality, with the average score being $99 \%$ on each assignment. Five quizzes, which tested their knowledge of various communication techniques, were administered on the Canvas learning management system at various time points throughout the semester (Table I). The average on the quizzes was $98 \%$, with the majority of students receiving $100 \%$ on each quiz.

At the end of the course, students also participated in an objective clinical structured exam (OSCE) which comprised of three stations 1) Device teaching (inhaler); 2) Patient interview and assessment/plan; and 3) Prescription drug counselling. Breakout rooms with TA were used for the OSCE stations. TAs used a grading rubric to assess student performance. The average score on the OSCE was 93\% (range $72 \%-100 \%$ ).
Students also completed a pre/post attitudes survey measuring their level of preparedness on a 5-point Likert scale, with 1 being "not at all prepared" to 5 being "very well prepared" (Table II). The greatest improvement was noted on student rating of their preparation for "using the SBAR to communicate with a physician" and "assess and address patient adherence issues" while the lowest gain was noted on the item "conducting an OTC medication consult" which is likely due to the fact they just completed OTC-focused skills course the previous semester. To provide a robust detection of the mean difference between the pre/post attitudes survey, both a parametric test and a nonparametric test were performed. For the parametric test, a two-tailed paired t-test was utilised. For the nonparametric test, the Mann-Whitney $U$ test was utilised as it is widely used when the dependent variable is ordinal but not normally distributed.

For all the questions on the preparedness survey, both paired t-test and Mann-Whitney $U$ test rejected the null hypothesis that the students reported the same level of preparedness before and after the course. Statistically significant improvements were noted in the scores across all 12 questions on the attitudes survey (Table II).

Table II: Pre/post attitudes survey measuring student level of preparedness ( $N=188$ )

\begin{tabular}{|c|c|c|c|}
\hline Rate your level of preparation for...* & Pre-mean & Post-mean & Absolute change \\
\hline Using the SBAR to communicate with a physician & 2.2 & 4.03 & 1.8 \\
\hline Assessing and addressing patient adherence issues & 2.6 & 4.28 & 1.67 \\
\hline Completing a medication reconciliation & 2.45 & 4.09 & 1.63 \\
\hline Addressing vaccine hesitancy & 2.48 & 4.11 & 1.62 \\
\hline Conducting a full medication history & 2.88 & 4.32 & 1.49 \\
\hline Assessing and addressing drug related problems (DRP) & 2.56 & 4.02 & 1.46 \\
\hline Interviewing a patient and take a full history & 2.88 & 4.32 & 1.44 \\
\hline Writing a SOAP note & 3.01 & 4.37 & 1.36 \\
\hline Teaching patients to use their inhalers & 3.09 & 4.36 & 1.27 \\
\hline Managing difficult conversations & 2.75 & 4.02 & 1.27 \\
\hline Counselling a patient on a new prescription & 3.28 & 4.31 & 1.03 \\
\hline Conducting an OTC medications consult & 3.29 & 4.22 & 0.94 \\
\hline
\end{tabular}

All items had a $p$-value $<0.001$

SBAR = situation background assessment and recommendation; OTC= Over the counter

*Level of preparedness on a five-point Likert scale of one being "not at all prepared" and five being "very well prepared."

Student's satisfaction with various components of the course was measured through a five-item survey. Overall, the students were satisfied with the course, with the highest satisfaction noted on the item "feedback provided by the TA"(Table III). Descriptive statistics using Excel was used for the satisfaction survey. 
Table III: Student satisfaction with various components of the course ( $N=197)$

\begin{tabular}{|c|c|c|c|c|c|}
\hline Course component & $\begin{array}{l}\text { Very satisfied } \\
\text { N (\%) }\end{array}$ & $\begin{array}{l}\text { Satisfied } \\
\text { N (\%) }\end{array}$ & $\begin{array}{c}\text { Neutral } \\
\text { N (\%) }\end{array}$ & $\begin{array}{c}\text { Dissatisfied } \\
\mathbf{N}(\%)\end{array}$ & $\begin{array}{c}\text { Very dissatisfied } \\
\mathbf{N}(\%)\end{array}$ \\
\hline Lecture & $621(45 \%)$ & $578(42 \%)$ & $144(10.5 \%)$ & $8(0.6 \%)$ & $30(2.1 \%)$ \\
\hline $\begin{array}{l}\text { TA portrayal of the } \\
\text { patient/physician }\end{array}$ & 869 (62.9\%) & $379(27.4 \%)$ & $88(6.3 \%)$ & $13(0.9 \%)$ & $32(2.3 \%)$ \\
\hline $\begin{array}{l}\text { Feedback provided by } \\
\text { the TA }\end{array}$ & 916 (66.3\%) & $356(25.8 \%)$ & 65 (4.7\%) & $13(0.9 \%)$ & $32(2.2 \%)$ \\
\hline $\begin{array}{l}\text { YouTube videos } \\
\text { depicting the consult }\end{array}$ & $853(61.8 \%)$ & $386(27.9 \%)$ & $110(7.9 \%)$ & $16(1.16 \%)$ & $16(1.16 \%)$ \\
\hline Overall simulation & 720 (52\%) & $490(35 \%)$ & $121(8.8 \%)$ & $20(1.44)$ & $30(2.2)$ \\
\hline
\end{tabular}

Numbers based on student satisfaction per simulation session (1381 responses were collected)

TA=Teaching assistant

\section{Future plans and recommendations}

This study suggests that a telehealth training model can be effective in teaching pharmacy students both technical skills and communication techniques necessary for practice in the ambulatory care setting. Trends in practice show that the use of telehealth modalities is on the rise (Klug et al., 2011; Traynor, 2013; Perdew et al., 2017; Badowski et al., 2018; Litke et al., 2018). While this course was borne out of necessity, plans include continuing the focus on using telehealth principles to train students in preparation for practice in the ambulatory care setting. Significant improvement was noted in student's ability to conduct a patient interview and address key issues such as vaccine hesitancy and drug related problems. Key skills such as using the SBAR tool had the highest gains (1.8) but lower overall level of preparation (4.03/5), indicating that there is a need for more practice in these areas. Future plans include the inclusion of SBAR training in other skills and interprofessional education (IPE) coursework.

Some limitations of the course design were noted, and key improvements can be made for future course offerings. This course utilised several modalities; however, further instruction on remote monitoring tools such as home blood pressure, blood glucose readings, food logs, and international normalised ratio (INR) results, among others, would be a useful addition to this course. In addition, based on the FIP 2021 report, it is clear that the inclusion of digital health tools such as mobile applications, artificial intelligence tools, blockchains, and bots will push the pharmacy profession into the new digital era (FIP, 2021). Adding some instruction on these key areas of digital health are the next steps for propelling the students into the future. Additionally, this course utilised student TAs, the use of standardised patients (SPS), which may provide a more realistic feel and have a greater impact on student learning.

Assessments in the course included the use of assignments, quizzes, and one OSCE. Additional and more frequent OSCEs would provide students more opportunities to perform in high stake situations and receive valuable feedback. Overall, the objectives for this skills class were met using this course design, and although some hands-on experiences had to be postponed to a future time (physical assessment), the authors felt that ambulatory care skills could be taught and assessed in a telehealth-based environment.

\section{References}

American Telemedicine Association (ATA, 2017). About telemedicine. Available at https://www.americantelemed.org/resource/whytelemedicine/

Badowski, M.E., Walker, S., Bacchus, S., Bartlett, S., Chan, J., Cochran, K.A., Coon, S., Liedtke, M., Phillips, B.G., \& White, T. (2018). Providing Comprehensive Medication Management in Telehealth. Pharmacotherapy, 38(2), e7e16. https://doi.org/10.1002/phar.2071

Healthy People 2020. Available at www.cdc.gov/nchs/healthy_people/hp2020.htm

International Pharmaceutical Federation (FIP). The FIP Development Goals: Transforming global pharmacy. The Hague: International Pharmaceutical Federation, 2020

International Pharmaceutical Federation (FIP). FIP Digital health in pharmacy education. The Hague: International Pharmaceutical Federation, 2021

Klug, C., Bonin, K., Bultemeier, N., Rozenfeld, Y., Vasquez, R. S., Johnson, M., \& Cherry, J.C. (2011). Integrating telehealth technology into a clinical pharmacy telephonic diabetes management programme. Journal of diabetes science and technology, 5(5), 1238-1245.

https://doi.org/10.1177/193229681100500533

Litke, J., Spoutz, L., Ahlstrom, D., Perdew, C., Llamas, W., \& Erickson, K. (2018). Impact of the clinical pharmacy specialist in telehealth primary care. American journal of health-system pharmacy, 75(13), 982-986. https://doi.org/10.2146/ajhp170633

Perdew, C., Erickson, K., \& Litke, J. (2017). Innovative models for providing clinical pharmacy services to remote locations using clinical video telehealth. American journal of 
health-system pharmacy, 74(14), 1093-1098. https://doi.org/10.2146/ajhp160625

Traynor K. (2013). Telepharmacy services bring new patient care opportunities. American journal of health-system pharmacy, 70(7), 565-566.

https://doi.org/10.2146/news130025

Vyas, D., Galal, S.M., Rogan, E.L., \& Boyce, E.G. (2018). Training students to address vaccine hesitancy and/or refusal. American journal of pharmaceutical education, 82(8), 6338. https://doi.org/10.5688/ajpe6338

Yemm, K.E., Arnall, J.R., \& Cowgill, N.A. (2020). Necessity of pharmacist-driven nonprescription telehealth consult services in the era of COVID-19. American journal of healthsystem pharmacy, 77(15), 1188.

https://doi.org/10.1093/ajhp/zxaa162

\section{Appendix I: Example of a teaching assistant (TA) character biography and script}

Purpose of the simulation: To train students to assess and address patient adherence.

\section{Character Biography:}

You are a 35-year-old HIV positive patient who was diagnosed with AIDS last year. Since then, you have been taking four HIV drugs and one antibiotic, which you have never filled. You are frustrated with life and feel a little depressed. You have lost several friends to AIDS and this has caused a lot of stress in your life.

Body language: Low energy level. Not happy or enthusiastic.

TA, please choose a student for case 1:

Read the following to the students:

"You have 10 minutes to make a plan for your patient. Please go to the electronic health record and review the patient's chart. For the HIV medications (darunavir, tenofovir/emtricitabine, cobicistat) and trimethoprim/sulfamethoxazole DS assume they are all needed for managing this patient's AIDS. Other medications can be changed, if needed."

Script:

\section{If asked how you are doing:}

TA: I am doing fine given the circumstances

If asked how your meds been working for you:

TA: Ok I guess. I have not noticed any improvements really If asked about side effects:

TA: I have been experiencing diarrhea a few times a week, which has been annoying

If asked what you are taking the senna for:
You: I am not sure

If asked whether you take your medications regularly:

TA: I am trying my best, obviously but sometimes I do not take my meds regularly. Frankly, I do not think it makes much of a difference but I guess I could do better.

If asked how often you miss a dose of your medications:

TA: I would say I miss one or two doses per week, may be more.

If asked about ways you could improve your medication adherence:

TA: I guess I could take my meds as prescribed by my doctor

If asked about reasons that you are unable to take your medications on time:

TA: Honestly, sometimes I just forget. It is hard to take so many meds.

If asked reason for not filling the trimethoprim/sulfamethoxazole:

TA: I guess I forgot to pick it up.

If asked about your financial circumstances:

TA: I have been having some difficulty affording my medications, I mean these drugs are really expensive but my health insurance helps a lot. The copay is 40 dollars per month but I would rather use that money for something fun.

If asked about other reasons for not taking your meds consistently:

TA: I just do not see the point, my friends have all died from AIDS and it is only a matter of time that I will also succumb.

If asked some good things about taking your medications on time:

TA: Well, my doctor did say my health would be better

\section{If asked to elaborate:}

TA: Well she said that getting my viral load under control will reduce the risk of infections and I will live longer. I have a son and I would like to see him grow up. Several of my friends died terrible deaths from opportunistic infections and it was terrifying.

If asked about next steps for improving your adherence:

TA: I guess I need to just start taking my meds as prescribed by my doctor.

If asked about strategies you use to remind yourself to take you medications:

TA: I do not use any tools to remind myself to take my medications

If asked what would work for you:

TA: I am pretty good on my phone, so perhaps I should try something on my phone 
If asked about your readiness for adhering to your medication regimen:

TA: Well probably a seven

If asked why a seven and not a one:

TA: Honestly, I really do need to get my health under control and I do not want to die from an infection

If asked what would have to happen for it to be an eight or nine:

TA: I think having someone to talk to will help.

If asked if anyone comes to mind:

TA: Not really, all my friends have died.

Student may recommend group therapy.

TA: That is a great idea. I need some support for sure.

If asked on a scale of one to ten, how confident you feel about making these changes?

TA: Honestly, I am at a nine. I really do think that your suggestions will help. Thank you for your help.

End the simulation. 